\title{
Evaluation de la formation dans le cycle de préparation à l'Agrégation des Sciences de la Vie et de la Terre au Maroc
}

\author{
El Mostafa Amiri, \\ Mustapha El Alaoui, \\ Rachid Janati-Idrissi, \\ Rajae Zerhane, \\ Mourad Madrane, \\ Mohamed Laafou,
}

Laboratoire Interdisciplinaire de Recherche en Ingénierie Pédagogique (LIRIP), Université Abdelmalek Essaadi, Ecole Normale Supérieure, Tétouan, Maroc

\section{Résumé}

Dans l'esprit d'enrichir et d'améliorer la qualité de l'enseignement des Sciences de la Vie et de la Terre au Maroc, un accord a été conclu entre le Maroc et la France en 1990 pour instaurer le cycle de préparation au concours de l'Agrégation, afin de recruter des professeurs agrégés de haut niveau scientifique, pédagogique et didactique. Toutefois, depuis l'instauration de ce concours, le pourcentage de réussite globale des candidats n'a jamais dépassé $16,8 \%$ dans toutes les promotions. L'analyse des rapports du jury du concours soulève des problèmes d'ordre cognitif et méthodologique auxquels sont confrontés les candidats. Pour résoudre ces problèmes, aucune étude de l'évaluation de la formation dans le cycle de préparation à l'agrégation n'a été réalisée; le document officiel ne renvoie nulle part à l'amélioration de la qualité de formation, mais touche seulement les programmes et les filières d'études. De plus, le rapport élaboré annuellement par le jury du concours, à caractère descriptif, peut induire une interprétation erronée des résultats. Pour tenter de trouver des explications à ces propos, une analyse multiple a été menée pour se renseigner sur la spécificité des épreuves du concours, les compétences recommandées par le jury, les contenus des cursus de préparation au concours et les pratiques de leur enseignement. Les résultats montrent que les enseignements des cursus de préparation à ce concours, marqués par des cours magistraux, ainsi que leur pratique d'enseignement, qualifiée de dogmatique, ne sont pas alignés avec les compétences scientifiques et 
pédagogiques requises pour le concours. En ce sens, la formation à l'Agrégation doit se détacher de la formation universitaire classique et proposer des projets de formation alternative innovants, répondant aux exigences du concours de l'Agrégation des Sciences de la Vie et de la Terre.

Mots clés: Agrégation des Sciences de la Vie et de la Terre, Résultats du concours, évaluation de la formation, cursus universitaire

\title{
Evaluation of Training in the Preparation Cycle for the Aggregation of Life and Earth Sciences in Morocco
}

\author{
El Mostafa Amiri, \\ Mustapha El Alaoui, \\ Rachid Janati-Idrissi, \\ Rajae Zerhane, \\ Mourad Madrane, \\ Mohamed Laafou,
}

Laboratoire Interdisciplinaire de Recherche en Ingénierie Pédagogique (LIRIP), Université Abdelmalek Essaadi, Ecole Normale Supérieure, Tétouan, Maroc

\begin{abstract}
In 1990, Morocco and France make an agreement to enrich and improve the quality of education of Life and Earth Sciences, and to establish the cycle of preparation for the competition of the Aggregation in order to recruit associate professors of high scientific, educational and didactic levels. However, since the creation of this competition in Morocco in 1991, the overall success rate of candidates has never exceeded $16.8 \%$ in all promotions. The analysis of the reports of the jury of the competition raises problems of a cognitive and methodological nature faced by the candidates. In order to solve the problems posed by this competition, no study of the evaluation of training in the cycle of preparation for aggregation has been carried out. The official document does not refer anywhere to improving the quality of training, but only to programs and courses of study. Moreover, the report drawn up annually by the selection board of the competition, which is of a descriptive nature, may lead to a misinterpretation of the results. In an attempt to find
\end{abstract}


explanations for this, a multiple analysis was carried to learn about, the specificity of the competition tests, the skills of recommended by the jury, the content of the competition preparation courses and their teaching practice. The results show that the lessons of the preparation courses for this competition, marked by lectures, and their teaching practice, qualified as dogmatic, are not aligned with the scientific and pedagogical skills of the competition. In this sense, the training in Aggregation must be detached from classical university training, and propose innovative alternative training projects meeting the requirements of the competition of the Aggregation of Life and Earth Sciences

Keywords: Aggregation of Life and Earth Sciences, Competition Results, Evaluation of Training, University Curriculum

\section{Introduction}

Les essais fournis pour améliorer l'enseignement des Sciences de la Vie et de la Terre se trouvent confrontés à des problèmes multiples ; les retombées de cet enseignement sont limitées par rapport aux buts assignés (Giordan, 1978 ; Désautels, 1980; Astolfi \& al, 1984 ; Giordan \& al, 1987 ; Madrane \& al, 2007). Le souci d'améliorer cet enseignement est un objectif prioritaire pour tout pays, car l'enseignement des sciences occupe une place de choix comme levier (Hulin, 1992). Il convient de souligner que dans ce système d'enseignement des sciences, l'enseignant joue un rôle important, car il est le seul à pouvoir concrétiser des projets d'enseignement en actions éducatives effectives et efficaces. Le développement de ces compétences disciplinaires et didactiques passe par la formation à l'enseignement des sciences, formation qui mérite un investissement important en termes de recherches et de budgets.

C'est dans cet esprit qu'un accord a été conclu entre le Maroc et la France pour instaurer le cycle de l'Agrégation des Sciences de la Vie et de la Terre. Ce concours se compose de quatre épreuves écrites (biologie et physiologie cellulaires, biologie et physiologie animales, biologie et physiologie végétales et géologie) et de trois épreuves orales (leçons propédeutiques de biologie, de géologie et de travaux pratiques de biologie) évaluant respectivement l'excellence des connaissances scientifiques de haut niveau et l'expertise professionnelle didactique et pédagogique. Si les épreuves écrites évaluent les compétences des candidats pour traiter les concepts scientifiques dans différents champs disciplinaires, les épreuves orales évaluent les compétences méthodologiques des candidats pour concevoir un plan d'étude cohérant, structuré, argumenté et présenté dans un délai de temps précisé par le jury du concours.

Toutefois, depuis la création de ce concours au Maroc, le pourcentage général de réussite n'a jamais dépassé 16,8\%, toutes promotions confondues. 
Malgré ces faibles résultats enregistrés d'une année à l'autre et les dysfonctionnements répétés, aucune recherche n'a été commandée par les instances officielles pour découvrir les causes et les obstacles qui entravent le déploiement d'actions efficaces de formation. Le document officiel, purement quantitatif, ne renvoie nulle part à l'amélioration de la qualité de la formation, mais se limite à la proposition de programmes, de curriculums et des filières d'étude. Il est en de même des rapports du jury du concours, purement descriptifs, qui ne tiennent nullement compte de la relation entre le curriculum réalisé et le contenu de l'évaluation des compétences cognitives et méthodologiques visées.

L'évaluation dans le champ de la formation a été considérée, dès les années 1980, comme une recherche d'information en fonction d'une grille de lecture, d'interprétation et de prise de décision (Barbier, 1985; De Ketele, 1986; Hadji, 1989, 1997 ; Stufflbeam, 1980). L'évaluation de la formation professionnelle doit viser d'une part les apprentissages réalisés, et d'autre part les compétences-clés qui contribuent à la réussite professionnelle. Selon Campagnale et Raiche (2008), l'évaluation explore les domaines de la notation, la définition de l'évaluation et de ses fonctions. Trois modèles se sont dégagés des recherches développées relativement à la problématique de l'évaluation: l'évaluation comme mesure; l'évaluation comme gestion d'apprentissage (Bloom, 1971; Cardinet, 1986; Scallon, 1988) et l'évaluation comme problématique du sens (Bonniol \& al, 1997). L'évaluation comme gestion des apprentissages est une évaluation formative, contrairement à l'évaluation comme problématique de sens, qui est plutôt un travail d'élucidation des phénomènes qui se produisent au cours du processus enseignement-apprentissage.

En ce qui concerne les objets de l'évaluation, il s'agit des produits de la formation (les apprentissages) et des processus à la base de l'apprentissage. Les fonctions de l'évaluation étaient pronostiques, formatives et sommatives (Allal \& al, 1979); actuellement, elles sont plutôt dites d'orientation, de régulation et de certification (Rogiers, 1997). Le réfèrent de l'évaluation est constitué de critères et d'indicateurs. L'évaluation du climat et de la qualité de la formation est une procédure nécessaire pour moduler et améliorer les actions de formation entreprises. Certains travaux publiés font appel à des questionnements par le biais desquels ils récoltent les perceptions des apprenants. Cependant, l'utilisation d'un questionnaire implique au préalable une validation visant à augmenter le caractère psychométrique du questionnaire utilisé. Il faut souligner que l'élaboration et la passation d'un questionnaire révèlent d'une méthodologie caractéristique du paradigme des processus médiateurs (Campanale \& al, 2008). 


\section{Problématique}

Le cycle de préparation à l'Agrégation des Sciences de la Vie et de la Terre, à l'instar de la France, a été installé au Maroc pour résoudre les problèmes liés à l'enseignement des Sciences de la Vie et de la Terre. Malgré un taux de réussite de plus en plus faible au cours des ans, le système de formation à l'Agrégation n'a été ni évalué, ni remis en cause pour augmenter l'efficacité des actions de formation. A l'exception des rapports sommaires élaborés par le jury du concours de sortie, aucune évaluation rigoureuse n'a été entreprise pour cerner les causes et les obstacles qui expliquent la régression des résultats dans ce cycle de formation. Les changements relatifs à la formation sont rares et ponctuels ; les reformes touchent principalement les programmes, les filières et les aspects institutionnels ; les réformes à des fins pédagogiques et opérationnelles sont rares. Les enseignants appliquent à la lettre les programmes figurant dans les cursus de formation, reconnaissent l'utilité de l'évaluation et souhaitent que l'institution s'en saisisse pour mettre en place des actions concrètes d'amélioration de l'enseignement (Bernard \& al, 1989). De tels programmes n'aboutissent pas à des enseignements efficaces s'ils ne sont pas transformés en activités pédagogiques cohérentes et placées dans un cadre d'étude productif.

Depuis la création du cycle de formation à l'Agrégation en 1991, l'évaluation développée s'inscrit essentiellement dans une perspective docimologique, avec une attestation des apprentissages. Elle n'a pas pour objet l'évaluation des pratiques de formation ni la compréhension des problèmes des apprentissages. Une telle évaluation est à mettre en relation avec des objectifs de certification et de sélection. Plusieurs questions clés sont totalement occultées, à savoir:

- La question des causes des dysfonctionnements et de la qualité de la formation dans le cycle de préparation à l'Agrégation ;

- L'adéquation entre le contenu de l'évaluation et le contenu de la formation ;

- La question de l'amélioration du système de formation et de préparation au concours national de l'Agrégation des Sciences de la Vie et de la Terre.

\section{Méthodologie}

Pour appréhender les propos des éléments de réponse aux questions de notre problématique, et partant du fait que la formation à l'enseignement des sciences est un système à plusieurs dimensions, plusieurs voies d'exploration sont donc possibles. En effet, vouloir approcher les paramètres qui conditionnent le climat et la formation à l'enseignement renvoie à une entreprise complexe, du fait du nombre important de facteurs susceptibles d'influencer le contexte et la qualité de la formation. Des approches 
renouvelées et des problématiques à construire seraient nécessaires dans l'analyse du système de formation à l'Agrégation, afin d'appréhender et de décrire, d'un coté, les différentes dimensions des pratiques de formation, et de l'autre coté, pour mettre en évidence les décalages de fonctionnement et d'ajustement réciproques des différents acteurs dans la situation de formationapprentissage. Ces acteurs sont:

- Les observations du jury du concours, soulignées dans les rapports de correction des épreuves ;

- Les sujets du concours, dont l'analyse permettra d'en mesurer la compatibilité avec les finalités soulignées par les instances officielles ;

- Les pré-acquis des candidats, pour évaluer leur alignement avec les exigences du concours d'Agrégation ;

- Les enseignements des cursus universitaires et du cycle de préparation à l'Agrégation, dont l'évaluation permettra de savoir s'ils pourraient constituer un socle de préparation solide au concours de l'Agrégation ;

- Les pratiques de l'enseignement, pour juger leur compatibilité avec les exigences du concours de l'Agrégation.

\section{Résultats et discussion}

\subsection{Un discours sur la formation : les rapports élaborés par les membres du jury}

Pour chaque promotion du concours, le jury rédige un rapport qui présente à la fois les résultats définitifs et des remarques d'ordre général sur les difficultés auxquelles sont confrontés les candidats. Le concours se compose de :

- Quatre épreuves écrites d'admissibilité de biologie et physiologie cellulaires, biologie et physiologie animales, biologie et physiologie végétales et de géologie; évaluant les compétences de connaissances scientifiques de haut niveau scientifique à travers des sujets de synthèse puisant des connaissances dans différents champs disciplinaires

- Trois épreuves orales d'admission, dont une leçon propédeutique de biologie (cellulaire, animale ou végétale), une leçon propédeutique de géologie, et de travaux pratiques de biologie. Ces activités évaluent l'expertise professionnelle, pédagogique et didactique des candidats. L'analyse des moyennes générales d'admissibilité et d'admission (graphe 1) dans toutes les promotions du concours montre que: 


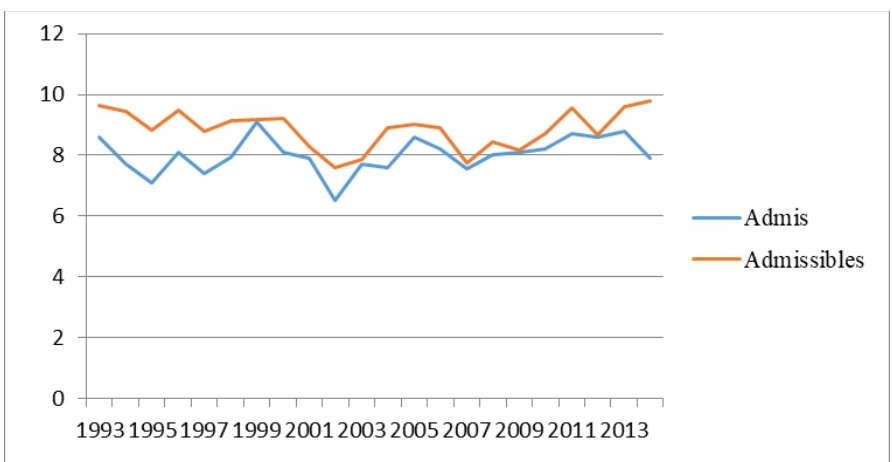

Graphe 1 : Évolution des moyennes générales des admissibles et des admis dans les promotions du concours

- Les deux moyennes d'admissibilité et d'admission sont faibles et à évolution synergique, elles traduisent des difficultés communes chez les candidats de toutes les promotions. Selon le jury du concours, les candidats n'ont ni les connaissances scientifiques générales, ni la méthodologie pour traiter les sujets du concours ;

- Les épreuves orales d'admission contribuent négativement aux résultats définitifs de ce concours; toutes promotions confondues, la moyenne générale des admissibles est toujours inférieure à celle des admis. Selon le jury, les résultats obtenus soulignent des difficultés d'ordre méthodologique, auxquelles sont confrontés les candidats, et qui seraient reflétées par des pratiques d'enseignement classiques.

Le rapport élaboré annuellement par le jury est essentiellement descriptif ; son caractère simpliste présente à nos yeux le risque et le danger d'une interprétation erronée, dans la mesure où il ne tient pas compte de la relation entre le curriculum réalisé et le contenu de l'évaluation d'une part, et des facteurs du contexte de formation d'autre part. Les rapports élaborés au fil des années se limitent à des descriptions et à des évocations, laissant au lecteur de procéder aux analyses et aux interprétations qui lui semblent pertinentes. Ces rapports comportent des remarques sur le niveau des candidats, des descriptions de faits et des conclusions. Des rapports susceptibles de fournir des indications pour l'amélioration de l'enseignement et de la formation doivent comporter:

- Des évaluations rigoureuses des apprentissages par rapport à un cadre référentiel ;

- Des analyses de pratiques et des programmes de formation ;

- Des stratégies et des activités de formation alternatives, utilisables par les formateurs. 


\subsection{Des sujets de l'écrit incompatibles avec les compétences visées par le concours}

Selon les instances officielles, la finalité du concours d'Agrégation des Sciences de la Vie et de la Terre est la sélection des professeurs agrégés de haut niveau scientifique, pédagogique et didactique. Cette élite de professeurs devrait être capable de traiter les concepts scientifiques dans différents champs disciplinaires et dans un cadre d'étude structuré, cohérent et argumenté. De telles compétences ne peuvent s'évaluer que par des sujets de synthèse, à approche d'étude pluridisciplinaire, qui obligeant les candidats à mobiliser des connaissances multiples couvrant l'aspect général de l'étude des concepts en question. L'approche d'étude pluridisciplinaire est facilitée par le rapprochement des disciplines et s'articule autour de l'unité essentielle, des divers domaines et objets de la science, qui constitue le principe épistémologique de son fondement (R. Dumonen \& al, 1996).

Toutefois, l'analyse des sujets des épreuves du concours souligne des contradictions avec les objectifs visés par les instances officielles. En effet, presque $50 \%$ des sujets des épreuves écrites de biologie et physiologie cellulaires, de biologie et physiologie végétales et de géologie (graphe 2), ont un aspect d'étude mono disciplinaire évaluant les candidats dans des compétences singulières. Ce type de sujet se limite à une restitution de connaissance qui ne reflète en aucun cas la culture scientifique générale qui marque le profil des professeurs agrégés.

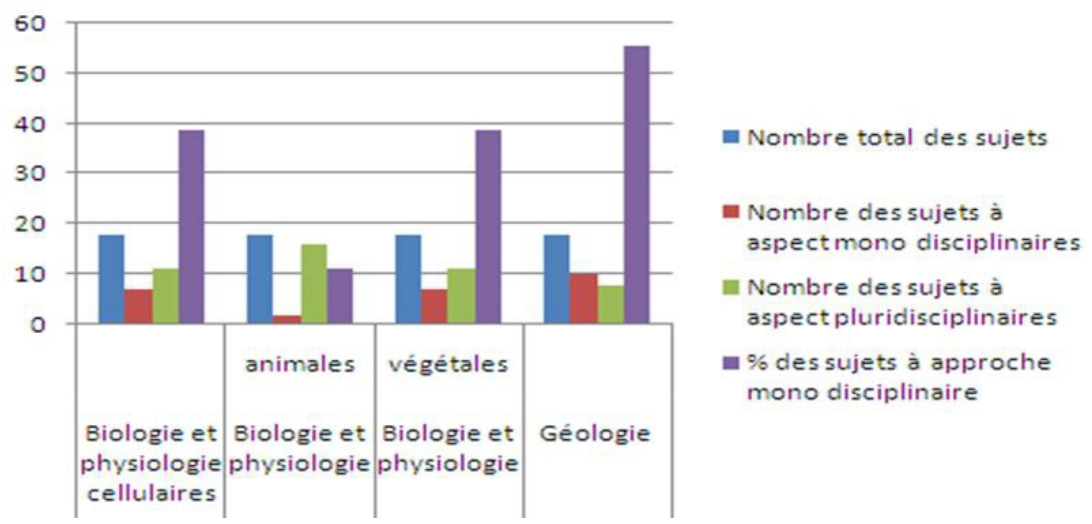

Graphe 2 : Catégorie des sujets des épreuves écrites du concours d'Agrégation des sciences de la Vie et de la Terre

\subsection{Les pré-acquis des candidats influent sur le résultat du concours}

Selon les instances officielles, le concours d'accès au cycle de préparation à l'Agrégation des Sciences de la Vie et de la Terre est ouvert à des candidats au profil de formation varié. Dans le but d'établir une éventuelle relation entre le profil de formation antérieure des candidats et les résultats définitifs du concours, nous avons comparé les résultats du concours de la promotion 1993 avec le profil des candidats de cette promotion (résultats du 
graphe 3). L'analyse de ces résultats montre que les candidats au profil de formation générale (titulaires de diplômes des écoles normales supérieures à formation scientifique générale) sont les plus favorisés pour réussir le concours d'Agrégation. Les candidats au profil de formation spécialisée (titulaires de licences spécialisées - biologie animale; biologie végétale et biologie générale -) présentent des résultats de réussite très moyens ; par contre, les candidats titulaires d'une licence de géologie et de DES sont incapables de réussir ce concours.

Ces résultats montrent que les candidats, au profil de formation spécialisée, sont mal préparés au concours de préparation à l'Agrégation des Sciences de la Vie et de la Terre, les enseignements de leurs cursus ne permettraient pas d'acquérir la culture scientifique générale exigée par ce concours.

Il en résulte que les instances officielles sont appelées à multiplier les licences de formation générale dans les universités marocaines, et à limiter l'accès à la formation au cycle de préparation à l'Agrégation aux candidats titulaires de ce type de licence.

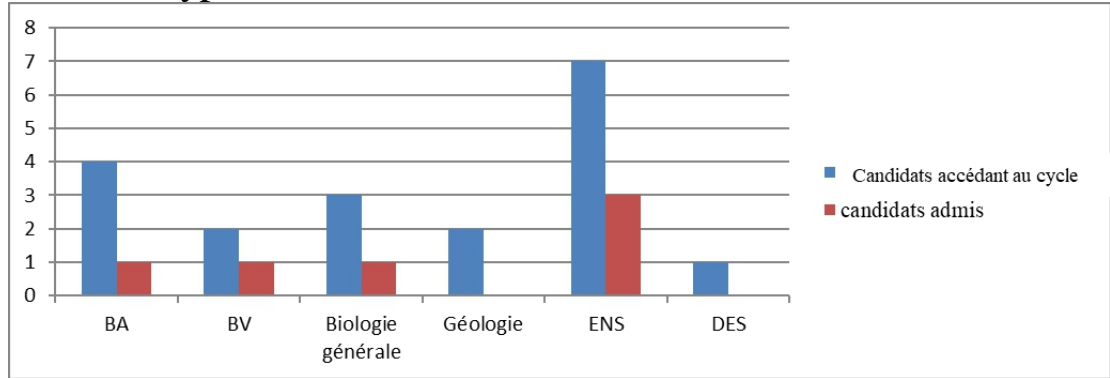

Graphe 3 : Relation profil des candidats-résultats du concours pour la promotion de 1993

3.4. Les cursus de préparation à l'Agrégation soulignent une spécialisation des enseignements, incompatibles avec les finalités du concours

Les cursus universitaires de l'enseignement des Sciences de la Vie et de la Terre ont été analysés pour mesurer leur alignement avec les exigences des épreuves du concours. Les enseignements de ces cursus sont organisés en systèmes modulaires aussi bien dans les universités que dans le cycle de préparation à l'Agrégation

\subsubsection{Organisation des enseignements des cursus universitaires}

L'analyse des cursus universitaires montre un cloisonnement et une spécialisation précoce des filières des enseignements:

- Entre sciences de la vie et sciences de la terre dès la deuxième année : seuls les semestres $\mathrm{S} 1$ et $\mathrm{S} 2$ fournissent des enseignements communs 
aux candidats, à partir du semestre 3, les enseignements sont divergents dans les deux filières en question;

- Entre les licences spécialisées dès la troisième année dans chacune de ces deux filières.

Ces cursus, très spécialisés, ne répondent pas aux exigences des sujets de synthèse de l'écrit du concours de l'Agrégation, qui obligent les candidats à mobiliser des connaissances dans différents champs disciplinaires. Les apports de cette analyse vont de pair avec les difficultés auxquelles sont confrontés les candidats lors des épreuves écrites d'admissibilité (résultats du graphe 1).

La spécialisation précoce des enseignements est renforcée par l'absence de licences spécialisées dans l'enseignement, de cours de pédagogie et de didactique des Sciences de la Vie et de la Terre. Il s'agit de contraintes curriculaires, qui sont à l'origine d'un manque d'expertise professionnelle pédagogique et didactique, révélant des difficultés d'ordre méthodologique chez les candidats, difficultés déjà mentionnées par le jury du concours.

\subsubsection{Organisation des enseignements du cursus du cycle de préparation à l'Agrégation des Sciences de la Vie et de la Terre}

Les enseignements du cursus du cycle de préparation à l'Agrégation des Sciences de la Vie et de la Terre sont organisés, comme dans le cas des cursus universitaires, en systèmes modulaires. La formation dans ce cycle, répartie sur deux années, est normalement conçue pour remplir les lacunes des candidats issus des universités. Par rapport aux cursus universitaires, le cursus du cycle de préparation à l'Agrégation présente des enseignements spécifiques à ce concours (leçons et travaux pratiques d'Agrégation) et des cours de didactique des sciences. Toutefois, les enseignements, dans ce cycle, sont dominés par les cours magistraux (résultats du graphe 4), qui sont en dichotomie avec les travaux pratiques; le volume horaire consacré à la préparation au concours de l'Agrégation (leçons et travaux pratiques d'Agrégation) est très réduit $(27 \%$ du volume horaire global); les enseignements de la didactique des sciences occupent un volume horaire qui représente $1 \%$ du volume horaire global. 


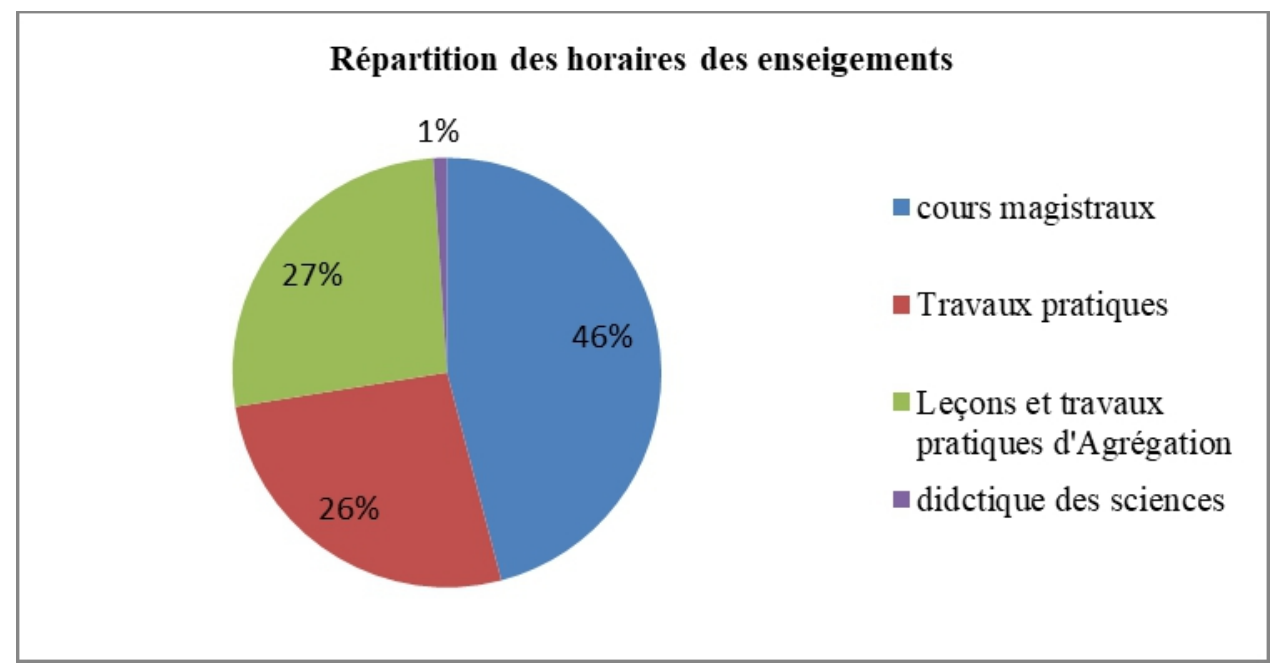

Graphe 4 : répartition du volume horaire des enseignements dans le cycle de préparation à l'Agrégation des Sciences de la Vie et de la Terre

Les enseignements du cursus de préparation à l'Agrégation des sciences de la vie et de terre sont spécialisés comme ceux des cursus universitaires. Ils présentent des connaissances cloisonnées et mono disciplinaires, qui ne permettent pas aux candidats de traiter les sujets dans différents champs disciplinaires. Il en résulte que ce cursus ne constitue, en aucun cas, une alternative pour surmonter les difficultés imposées par les sujets du concours.

Il en est de même pour les activités spécifiques de la préparation au concours de l'Agrégation (leçons et travaux pratiques d'Agrégation, didactiques des sciences), à volume très réduit, qui sont de ce fait insuffisantes à cerner les problèmes posés par le concours. Ces données concordent avec les observations faites par le jury quant au niveau des candidats au concours.

\subsection{Les pratiques de l'enseignement dans les universités et dans le cycle de préparation à l'Agrégation}

La méthodologie de l'enseignement est un facteur à prendre en considération dans la préparation aux épreuves du concours de l'Agrégation des Sciences de la Vie et de la Terre. Ces épreuves, de type synthèse, font appel à l'approche d'étude pluridisciplinaire, mobilisant des connaissances dans différents champs disciplinaires. Les connaissances scientifiques doivent se construire à partir de l'exploitation des données concrètes (phénomènes biologiques ou géologiques), à laquelle les candidats procèderont en faisant preuve d'esprit scientifique, en étant capables de présenter une problématique relative à l'étude du concept en question, de proposer des hypothèses explicatives, de concevoir des expériences pour vérifier l'authenticité de ces hypothèses, et enfin d'interpréter les résultats obtenus. 
Toutefois, les résultats de l'analyse des enseignements des cursus universitaires et du cycle de préparation à l'Agrégation soulignent une contradiction avec les finalités du concours. Les enseignements, dominés par des cours magistraux spécialisés, renvoient à la restitution des connaissances et non à leur construction par les candidats. Ils traduisent une pratique d'enseignement classique reflétant une pédagogie linéaire qui ne contribue en aucun cas au développement de l'esprit scientifique chez les candidats au concours.

En plus, les travaux pratiques sont en dichotomie avec les cours magistraux, ce qui ne permettra pas aux candidats d'intégrer l'expérimentation dans l'explication des phénomènes scientifiques. Cette dichotomie ne permet en aucun cas de présenter des productions structurées conçues sous forme d'une argumentation relative aux problèmes scientifiques à résoudre. Il s'agit de l'un des nombreux cas d'incompatibilité avec les finalités exigées par le concours, et qui ne sont pas prononcés par les instances officielles. Le manque d'une culture scientifique générale, conséquence des cours magistraux spécialisés, en dichotomie avec les travaux pratiques, est à l'origine des difficultés auxquelles sont confrontés les candidats au concours de l'Agrégation des Sciences de la Vie et de la Terre

Le manque de cours de pédagogie et de didactique, dans les cursus universitaires et leur insuffisance dans le cursus du cycle d'Agrégation, sont à l'origine de l'incapacité des candidats à convertir les programmes d'enseignement universitaires à des fins pédagogiques exploitables pour faire émerger de nouvelles connaissances scientifiques. Ces données soulignent des contraintes curriculaires qui sont à l'origine des difficultés d'ordre méthodologique auxquelles sont confrontés les candidats.

Plusieurs recherches ont entretenu une réflexion sur la pédagogie de l'enseignement aux universités: un enseignement universitaire efficace doit permettre le rapprochement des disciplines qui s'articulent autour de l'unité essentielle des divers domaines et objets de la science (Develay, 1996). L'interdisciplinarité est le fruit de l'association de disciplines diverses, qui permet l'émergence de nouveaux concept (Unesco, 1986). Il en résulte que le savoir disciplinaire, pris isolément, ne permettra pas de traiter d'une manière exhaustive les sujets de synthèse du concours de l'Agrégation des Sciences de la Vie et de la Terre. Selon El Hage (2011), l'enseignement universitaire qui veut être efficace doit dépasser l'exposé des contenus de cours; d'où la nécessité de changer sa façon d'enseigner en passant des pédagogies passives, traditionnelles, centrées sur l'enseignant et sur le contenu, à des méthodes innovatrices et actives centrées sur l'étudiant. 


\section{Conclusion}

Le but de cette recherche est d'évaluer la formation dans le cycle de préparation à l'Agrégation des Sciences de la Vie et de la Terre au Maroc. Cette évaluation nous semble constituer un besoin pour expliquer une vérité représentée par les résultats catastrophiques des candidats au fil des promotions du concours. L'analyse de plusieurs paramètres nous a semblé utile pour présenter des éléments d'une évaluation efficace de la formation à l'Agrégation:

- L'évaluation faite par le jury du concours est purement normative, où la performance d'un candidat est comparée à celle des autres candidats par un même instrument

- Les instances officielles se sont limitées à l'élaboration de programmes d'étude sans finalités pédagogiques ;

- Le jury du concours, sans cadre de référence, ne souligne que des observations d'ordre général, révélant des reproches concernant le faible niveau scientifique et méthodologique des candidats ;

- Presque 50\% des sujets de l'épreuve écrite du concours ont un aspect mono disciplinaire, et n'évaluent pas les compétences soulignées par les instances officielles ;

- Les formateurs sont incapables de concevoir des enseignements innovants, compatibles avec les compétences visées par le concours.

Notre étude a permis d'approcher les propos de ces résultats. Les finalités tracées par les instances officielles, les observations faites par le jury du concours, le contenu des enseignements des cursus de préparation au concours et les pratiques des enseignements, présentent un écart avec les compétences visées par le concours d'Agrégation. Si la qualité de synthèse, évaluée par les épreuves du concours, vise une sélection efficace de professeurs agrégés de haut niveau scientifique, pédagogique et didactique, ni les programmes des cursus universitaires et de préparation à l'Agrégation, ni les pratiques de leurs enseignements ne peuvent doter les candidats de compétences cognitives et méthodologiques susceptibles de les aider à surmonter les problèmes posés par le concours en question. Les enseignements universitaires et du cycle de préparation à l'Agrégation, marqués par une pédagogie linéaire, reflétée par des enseignements mono disciplinaires et spécialisés, ne permettent pas aux candidats, au profil de formation très hétérogène, de traiter les concepts scientifiques dans différents champs disciplinaires. En effet, la forme magistrale des cours et leur dichotomie avec les travaux pratiques sont à l'origine d'un savoir qui ne laisse aucune place aux candidats dans la construction et l'argumentation des notions scientifiques relatives aux sujets des épreuves du concours. Cette approche d'enseignement, qualifiée de classique, incitant à la restitution des connaissances, est 
incompatible avec les actions qui peuvent garantir le développement de réelles compétences pour l'exercice du métier d'enseignement. En effet, dans les cursus universitaires, les notions d'enseignements, cloisonnées et spécialisées, sont présentées dans un cadre d'étude incohérent, et ne contribuent en aucun cas à l'émergence des concepts unificateurs des connaissances scientifiques.

Nous pensons que malgré le caractère ponctuel et singulier de cette évaluation, les observations dégagées d'une interprétation adéquate des résultats permettraient d'améliorer la qualité de la formation dans le cycle de préparation à l'Agrégation des Sciences de la Vie et de la Terre. L'engagement, l'implication et la motivation d'un groupe de formateurs sont nécessaires pour engager des réflexions approfondies, visant à concevoir des actions de formation alternatives, qui puissent améliorer la portée et le climat de cette formation. Des échanges avec d'autres institutions internationales sont nécessaires pour instaurer une conduite de changement vers de nouvelles réflexions débouchant sur des pratiques de formation novatrices.

En ce sens, la formation à l'Agrégation doit se détacher de la formation universitaire classique et programmer des enseignements qui convergent vers la construction de concepts scientifiques unificateurs, rapprochant divers champs disciplinaires. Il y a lieu de souligner que l'investigation des pratiques de formation nécessite des protocoles et des méthodologies spécifiques qui seraient à construire. Ainsi, des évaluations fines et ciblées permettraient de mettre sur pied un nouveau plan stratégique, ainsi que les recentrages qui s'imposent, de temps à autre, dans certains de ses choix, en vue de mieux les adapter aux mutations rapides et profondes que connait notre environnement tant sur le plan économique que technologique, culturel et social. Ces évaluations seraient nécessaires pour suggérer des conditions pour l'instauration d'un processus d'implantation d'une réforme profonde du système de formation dans le cycle de préparation à l'Agrégation des Sciences de la Vie et de la Terre.

La préoccupation actuelle serait l'intégration des pratiques d'évaluation dans le processus de formation à l'Agrégation. Cependant, le passage de l'interprétation des données d'une évaluation aux implications pédagogiques n'est ni évident, ni simple, et requiert des compétences diverses, susceptibles de garantir la conception de procédés efficaces d'évaluation et d'exploitation optimale des données de l'évaluation dans une perspective d'amélioration des actions de formation.

\section{References:}

1. Allal, L. Cardinet, J. \& Perrenoud, P. (1979). L'évaluation formative dans un enseignement différencié. Berne: Peter Lang. 
2. Astolfi, J. P. Causiline-Marmàcèche, E. Giordan, A. HenriquesChristofidès, A. Mathieu, J. \& Weil-Barais, A. (1984). Expérimenter sur les chemins de l'explication scientifique. Toulouse: Privat.

3. Barbier, J.M. (1985). L'évaluation en formation. Paris : PUF.

4. Bernard, H. \& Trahan, M. (1989). Les principales composantes d'une politique d'évaluation de l'enseignement. Mesure et évaluation en éducation.

5. Bloom, B. (1971). Handbook on formation and summative evaluation of student learning. New York: Mc Graw-Hill Book Co.

6. Bonniol, J-J. \& Vial, M. (1997). Les modèles de l'évaluation: Textes fondateurs avec commentaires. Paris : De Boeck \& Larcier.

7. Campgnale, F. \& Raiche, G. (2008). L'évaluation dans la formation supérieure et professionnelle. Mesure et évaluation en éducation.

8. Cardinet, J. (1986). Pour apprécier le travail des élèves. Bruxelles : De Boeck.

9. De Ketele, J.M. (1986). L'évaluation: approche descriptive ou perspective? Bruxelles: De Boeck.

10. Désautels, J. (1980). Ecole+science= Ecgec.Québec, Québec.

11. Develay, M. (1996). Donner le sens à l'école, Paris, ESF.

12. Dumonen, R. \& Astolfi, J. P. (1996). Didactiques de Sciences de la Vie et de la Terre. Edition Nathan.

13. El Hage, F. (2011). L'enseignement des sciences à l'université: traditions ou innovations? Education et socialisation. [Online] :http://journals.openedition.org/edso/1785, DOI: 10.4000/edso.1785, (consulté : 25 février 2018).

14. Giordan, A. \& DE Vecchi. (1987). Les origines du savoir. NeuchâtelParis, delachaux \& niestlé.

15. Giordan, A. (1978). Une pédagogie pour les sciences expérimentales. Paris, Centurion.

16. Hadji, Ch. (1989). L’évaluation, règles du jeu. Paris: ESF.

17. Hadji, Ch. (1997). L'évaluation démystifiée. Paris : ESF.

18. Hulin, N. (1992). Une question lancinante : comment former à l'enseignement des sciences. In Sciences à l'école : les raisons du malaise, sciences et vie, $\mathrm{n}^{\circ} 180$.

19. Madrane, M. Khaldi, M. \& Talbi, M. (2007). Exploitation didactique de l'histoire des sciences dans une perspective de formation à l'enseignement des sciences. Didascalia-n'31. [Online]: http://documents.irevues.inist.fr/bitstream/handle/2042/23977/DIDA SKALIA_2007_31_167 pdf? Séquence (consulté le 06/12/2019)

20. Rogiers, X. (1997). Evaluer une action d'enseignement ou de formation. Bruxelles: De Boeck. 
21. Scallon, G. (1988). L'évaluation formative des apprentissages. Tome 1. La réflexion. Sainte-Foy, Quebec: Presses de l'Université de Laval. 22. Stufflebeam, D. (1980). L'évaluation en éducation et la peise de décision. Otawa: NHP.

23. Unesco, (1986). L'interdisciplinarité dans l'enseignement général. [Online]: http//www.org/education/pdf (consulté; 24/06/2019). 